\title{
Lernaufgaben und Rückmeldekultur in einem kompetenz- orientierten GW-Unterricht
}

\author{
*hanspeter.gottein@phsalzburg.at, Pädagogische Hochschule Salzburg - Stefan Zweig \\ eingereicht am: 01.02.2017, akzeptiert am: 21.08.2017
}

\begin{abstract}
Bildungsstandards und zentrale Reifeprüfung bedingen eine vermehrte Hinwendung zu einem kompetenzorientierten Unterricht. Per Definition sind Kompetenzen nicht direkt beobachtbar, sondern nur indirekt erschließbar, entsprechenden komplexen Aufgabenstellungen und qualitätsvollen Rückmeldungen kommt daher große Bedeutung zu.

Zunächst wird die Rolle von Lernaufgaben beschrieben, es folgt dann eine Aufarbeitung des Begriffs ,kompetenzorientierte Lernaufgabe‘. Anhand von Webbs DOK-Modell wird eine Möglichkeit zur Einschätzung von Komplexitätsgraden vorgestellt, Marzanos 4.0 Skala bietet Möglichkeiten der Leistungsrückmeldung.
\end{abstract}

Keywords: Aufgabenkultur, Lernaufgaben, Rückmeldekultur, Feedback

\section{Learning tasks and feedback culture in a competency-based GW education}

Educational standards and centralized graduation require an increased attention to a competency-based education. By definition, competences are not directly observable, but only indirectly ascertainable. Correspondingly, complex tasks and highquality feedback is therefore of great importance.

First, the role of learning tasks is described, followed by a reappraisal of the term "competency-based learning task". Based on Webb's DOK model, a way of assessing levels of complexity is introduced. Marzano's 4.0 scale provides opportunities for learning-performance feedback.

Keywords: task-based culture, learning tasks, feedback culture, feedback

\section{$1 \quad$ Hintergrund und Ziel des Beitrags}

Gegenwärtig können in vielen Bildungssystemen erhöhte Anstrengungen in Hinblick auf eine vermehrte Output-Orientierung festgestellt werden, erkennbar u. a. durch die Implementierung von Bildungsstandards sowie zentralen bzw. teilzentralen Zwischen-, Abschluss- und Diplomprüfungen. Die dabei im Zentrum stehenden Aufgabenstellungen testen meist kein replizierbares Wissen ab. Derartige Aufgaben verlangen Vernetzungs- und Transferleistungen, um gelöst werden zu können. Dies wiederum ist eine große Herausforderung für den Unterricht, denn dieser muss durch eine entsprechende Lern- und Aufgabenkultur geprägt sein, um die angesprochenen Vernetzungs- und Transferleistungen zu ermöglichen. Dass Unterricht jedoch auf diese Herausforderungen nur zögerlich eingeht, wird durch zahlreiche Studien belegt. So stellt etwa Astleitner (2007: 137) fest, dass der fragend-entwickelnde Unterricht immer noch die dominante Unterrichtsform darstellt. Hattie (2012: 72) berichtet mit Verweis auf eine Studie von Yair von Sprechanteilswerten von Lehrerinnen und Lehrern von 70-80\% der Unterrichtszeit in den Jahrgangsstufen 6-12, in höheren Jahrgangsstufen steigen diese Werte noch weiter an. Weiters verweist Hattie (2012: 75) auf Erkenntnisse von Brualdi, welcher an typischen Unterrichtstagen 200-300 Fragen von Lehrpersonen an Schüler/innen zählte; mehr als 60 \% dieser Fragen zielten dabei lediglich auf die Wiedergabe von Fakten ab. Dabei werden Transfer und Anwendung nicht gefördert, sondern oftmals „träges Wissen “ (Renkl 1994) oder „inert knowledge“ (Whitehead 1967) produziert. Dörner (1990: 304) spricht dabei sarkastisch von Eunuchenwissen: „Sie wissen, wie es geht, können es aber nicht“. Nötig wäre die Vermittlung intelligenten Wissens im Sinne von Franz Weinert. Solches ist „bedeutungshaltig und sinnhaft [...]. 
Gut verstandenes Wissen ist ein Wissen, das nicht eingekapselt ist, nicht tot im Gedächtnis liegt, nicht verlötet ist mit der Situation, in der es erworben wurde, sondern das lebendig, flexibel nutzbar, eben intelligent ist" (Weinert 2000, zit. n. Helmke 2009: 42).

Aus Platzgründen können hier weitere Studien nicht zitiert werden, eines zeigt sich jedoch bereits jetzt daraus: Der Förderung anwendungsfähigen Wissens (Handlungskompetenz), ermöglicht durch entsprechende Aufgabenstellungen, kommt in einem kompetenzorientierten Unterricht ein zentraler Stellenwert zu.

\section{Lernaufgaben in einem kompetenz- orientierten Unterricht}

Vorliegender Artikel fokussiert auf die Bedeutsamkeit von Lernaufgaben und einer im Zusammenhang damit stehenden Rückmeldekultur. Eine detaillierte theoretische Begründung der Relevanz von Lernaufgaben im Rahmen eines kompetenzorientierten Unterrichts würde den Rahmen dieses Artikels bei Weitem sprengen, dennoch ist es notwendig, eine Einordnung in aktuell anerkannte Modelle guten (kompetenzorientierten) Unterrichts vorzunehmen und die Begriffe ,Kompetenz', ,Lernaufgabe 'bzw. ,kompetenzorientierter und guter Unterricht' im Sinne dieser Abhandlung zu definieren. Im Anschluss daran wird eine wichtige Problematik eines kompetenzorientierten Unterrichts thematisiert: Per Definition zeigt sich Kompetenz nur indirekt über Handlungen. Dafür benötigt man entsprechende Aufgabenstellungen. Daraus ergeben sich wiederum zwei Kernfragen:

1. Wie bestimmt man die Komplexität von Aufgabenstellungen?

2. Wie bewertet man komplexe Leistungen?

Im pädagogischen Kontext wird der Begriff der Kompetenz unterschiedlich diskutiert und daher u. a. auch als „fuzzy concept“ (Boon \& van der Klink 2002, zit. n. Winterton, Delamare - Le Deist \& Stringfellow 2006: 29) bezeichnet. Hans Aebli (1980: 98) konkretisiert den Begriff etwas, indem er Kompetenzen vor allem im Sinne von Handlungen sieht, die entsprechende Wissensbasis (= Sachkompetenz) definiert er lediglich als notwendige Voraussetzung für kompetente Handlungen. Im pädagogischen Kontext hat sich vor allem die (psychologische) Definition von Weinert durchgesetzt, in der vor allem das „Problemlösen in variablen Situationen " (Weinert 2002: 28) in den Vordergrund gerückt wird. Wormeli (2006: 12) verweist auf das Mastery-Konzept und definiert wie folgt: "Students have mastered content when they demonstrate a thorough understanding as evidenced by doing something substantive with the content beyond merely echoing it. [...]; it's the masterful student who can break content into its component pieces [...]".

Um Schülerinnen und Schülern ein bewusstes Erleben der eigenen Kompetenz zu ermöglichen, ist Klarheit und Transparenz hinsichtlich der angestrebten Lernziele unerlässlich. Kompetenzorientierter Unterricht kann sinnvollerweise nur auf Basis von vorher festgelegten Zielen konzipiert werden, diese müssen den Schülerinnen und Schülern unbedingt transparent gemacht werden, um die drei Grundfragen der Reflexion über das eigene Lernen beantworten zu können: Was sind die Ziele? Wie/mit welchen Mitteln strebe ich nach diesen Zielen? Was ist der nächste Zwischenschritt? (vgl. Hattie 2012). Dieselbe Forderung stellt Rick Wormeli unter dem Schlagwort „Begin with the end in mind“ (2006: 21) auf und betont, dass dieses Nachdenken über das Ende (die Ziele des Unterrichts) sowohl aufseiten der Lehrperson(en) als auch aufseiten der Schüler/innen stattfinden muss, um kompetenzorientiertes Lernen zu ermöglichen.

Die unterschiedlichen Ansätze in der Kompetenzdiskussion weisen als Gemeinsamkeit auf, dass Kompetenzen aus Handlungen nicht direkt beobachtbar, sondern vielmehr nur implizit erschließbar sind. Dies erklärt die zentrale Bedeutung von Aufgabenstellungen im Rahmen eines kompetenzorientierten Unterrichts. Diese Bedeutung und die im Zusammenhang damit stehende Bedeutung einer entsprechenden Rückmeldung von Schüler/innen-Leistungen wird auch in verschiedenen Modellen guten Unterrichts betont. In Tabelle 1 werden die entsprechenden Erläuterungen aus verschiedenen Modellen zusammengestellt ${ }^{1}$. Herangezogen wurden dabei zwei Modelle aus dem deutschsprachigen Raum, die Merkmale guten Unterrichts nach Hilbert Meyer $(2004 ; 2010)$ und Andreas Helmke (2009: 168 ff.) sowie zwei Modelle aus dem angloamerikanischen Raum, die Konzeption nach Jere Brophy (2000) und John Hattie (2009; 2012).

Der Begriff der Lernaufgaben ist vor allem in Anschluss an das schlechte Abschneiden der Schüler/innen des deutschen Sprachraumes bei internationalen large-scale assessments ins Zentrum der pädagogischen Diskussion gerückt (z. B. Institut für Qualitätsentwicklung (Hessisches Kultusministerium 2011: 2). Die „empirische Wende“ (Helmke 2009: 16) im Anschluss an den ,PISA-Schock' führte zu einer vermehrten Output-Orientierung der deutschsprachigen Bildungssysteme, die sich u. a. durch die Implementierung von Bildungsstandards zeigten. Ein zentrales Element von Bildungsstandards ist der erwartete

\footnotetext{
Für eine ausführlichere Übersicht einer vergleichenden Gegenüberstellung von insgesamt sechs Modellen guten Unterrichts hinsichtlich aller Merkmale vgl. Gottein 2016: $55 \mathrm{f}$.
} 
Tab. 1: Merkmale guten Unterrichts hinsichtlich einer Aufgaben- und Rückmeldekultur

\begin{tabular}{|c|c|c|c|c|}
\hline & Meyer & Helmke & Brophy & Hattie \\
\hline Aufgaben & $\begin{array}{l}\text { Intelligentes Üben (dem } \\
\text { Lernstand entspre- } \\
\text { chende Aufgabenstel- } \\
\text { lungen), individuelles } \\
\text { Fördern }\end{array}$ & $\begin{array}{l}\text { Konsolidierung und } \\
\text { Sicherung des Unter- } \\
\text { richtsertrages durch } \\
\text { entsprechende Auf- } \\
\text { gabenstellungen, } \\
\text { kompetenzorientierter } \\
\text { Unterricht mit entspre- } \\
\text { chenden Aufgabenstel- } \\
\text { lungen }\end{array}$ & $\begin{array}{l}\text { Ausreichendes Ange- } \\
\text { bot an Übungs- und } \\
\text { Anwendungsaufgaben, } \\
\text { Förderung individueller } \\
\text { Entwicklungsstrukturen } \\
\text { durch ,scaffolding' } \\
\text { (individuell angepasste } \\
\text { Unterstützungsstruktur) }\end{array}$ & $\begin{array}{l}\text { Zielklarheit durch drei } \\
\text { Fragen: } \\
\text { - Was ist das Ziel? } \\
\text { - Wie kommen wir dort } \\
\text { hin? } \\
\text { - Was ist der nächste } \\
\text { Schritt? } \\
\text { Vernetzungsmöglichkeit } \\
\text { schaffen durch Konst- } \\
\text { ruktion, Dekonstruktion, } \\
\text { Rekonstruktion }\end{array}$ \\
\hline Rückmeldung & $\begin{array}{l}\text { Transparente Leistungs- } \\
\text { erwartungen im Sinne } \\
\text { eines, contracting' mit } \\
\text { Schülerinnen und Schü- } \\
\text { lern, inhaltliche Klarheit } \\
\text { durch transparente } \\
\text { Zielorientierung }\end{array}$ & $\begin{array}{l}\text { Lernförderliches Klima, } \\
\text { u. a. durch konstruk- } \\
\text { tiven Umgang mit } \\
\text { Fehlern, Trennung von } \\
\text { Lern- und Leistungssitu- } \\
\text { ationen }\end{array}$ & $\begin{array}{l}\text { Inhaltliche Klarheit } \\
\text { schaffen durch Zielklar- } \\
\text { heit und Vorausstruktu- } \\
\text { ren (Advance Organi- } \\
\text { zer) sowie Entwicklung } \\
\text { von Fragen und Fragen- } \\
\text { komplexen, die Diskurse } \\
\text { über die Unterrichtsin- } \\
\text { halte anregen sollen }\end{array}$ & $\begin{array}{l}\text { Fehler und Lernumwege } \\
\text { werden als Lernchance } \\
\text { wahrgenommen und } \\
\text { transparent gemacht, } \\
\text { Trennung von Lern- und } \\
\text { Leistungssituationen, } \\
\text { Feedback auf Basis stän- } \\
\text { diger, beurteilungsfreier } \\
\text { Lernstandserhebungen }\end{array}$ \\
\hline
\end{tabular}

Outcome (Performanz), welcher wiederum nur durch entsprechende Aufgabenstellungen ermittelt werden kann.

Eine wesentliche Erkenntnis im Zusammenhang mit dem erwähnten mangelhaften PISA-Abschneiden deutschsprachiger Schüler/innen war u.a., dass diese mit den dabei verwendeten komplexen und kompetenzorientierten Aufgabenformaten zu selten konfrontiert werden bzw. diese kleinschrittig gemeinsam mit Schülerinnen und Schülern in einem lehrpersonen-zentrierten Unterricht erarbeitet werden, da ein weiteres Merkmal guten Unterrichts - die hohe Erwartung an die Leistungsfähigkeit und -willigkeit der Schüler/innen - nicht zur Anwendung gebracht wird (vgl. Ziegler, Stern, \& Neubauer 2012: 22). Die Selbstbestimmungstheorie der Motivation nach Edward Deci und Richard Ryan (2002) zeigt auf, dass eine möglichst häufig vorkommende Bewältigung herausfordernder Situationen (Lernaufgaben) die Selbstwirksamkeit steigern und somit die eigene Kompetenz bewusst werden lässt.

Diese Ausführungen legen nahe, dass Lernaufgaben in einem kompetenzorientierten Unterricht ein zentraler Stellenwert zukommt. Keller und Reintjes (2016: 15) bezeichnen Aufgaben gar als „Schlüssel zur Kompetenz", für Parchmann und Bernholt (2016: 41) sind Aufgaben „Brücken zwischen Lebenswelt und Fachunterricht", Reinfried (2016: 4) bezeichnet Lernaufgaben als „Schlüsselbegriff für fachdidaktische Unterrichtsentwicklung". Was aber macht eine Aufgabe zu einer kompetenzorientierten Aufgabe?

\section{Was macht eine Lernaufgabe zu einer ,guten' (kompetenzorientierten) Aufgabe?}

Wie bereits einleitend erwähnt, ist ca. ab der Jahrtausendwende die Hinwendung zu einem kompetenzorientierten (output-orientierten) Unterricht eines der zentralen Elemente in der pädagogischen Diskussion. Im Zusammenhang mit der Implementierung von Bildungsstandards wird u. a. im Rahmen der KliemeExpertise eine empirisch valide Erfassung von Kompetenzen durch entsprechende Aufgabenstellungen gefordert, welche ,ausgehend von den Kompetenzbeschreibungen [...] prüfen, ob eine Person das angestrebte Ergebnis oder Handlungspotential entwickelt hat" (Klieme et al. 2007: 23). Problematisch ist in diesem Zusammenhang, dass nach wie vor keine einheitliche Definition des Begriffes ,Lernaufgabe' vorliegt (vgl. Reinfried 2016: 6). Eine umfassende Definition des Begriffes wurde von Kurt Reusser (2013) vorgelegt. Diese repräsentiert die Kernideen einer Lernaufgabe im Sinne der eingangs erwähnten PISAAufgaben.

Gute Lernaufgaben nach Reusser (2013: 5; Kürzung und Hervorhebungen durch den Autor)

- lenken den Blick der Lernenden auf jene Konzepte, Zusammenhänge, Prozesse, Fertigkeiten und Haltungen, die den Bildungsgehalt von Fächern auszeichnen [...];

- begünstigen individuelle Lern- und Bearbeitungswege auf unterschiedlichen Leistungsniveaus $[\ldots]$; 
- repräsentieren fachliche Kernideen [...];

- laden zu tiefem Verständnis und Problemlösen ein;

- trainieren und festigen Fertigkeiten und Strategien;

- stossen [sic!] situativ Kommunikations- und Kooperationsprozesse an;

- ermöglichen aktiv-entdeckendes und selbstgesteuertes Lernen [...];

- sind in einen sinnstiftenden Kontext eingebunden $[\ldots]$;

- wecken Neugier und Motivation [...];

- ermöglichen das Nachdenken und die Reflexion der Lernenden über die Welt und über ihr eigenes Lernen [...];

- sind adaptiv an das Vorwissen der Lerngruppe angepasste, gehaltvolle Aufgaben [...].

Wilkening (2016: 15f.) fasst Erkenntnisse aus der Feedbackforschung zusammen und streicht im $\mathrm{Zu}$ sammenhang mit Lernaufgaben folgende wichtige Maßnahmen heraus:

- hohe Erwartungshaltung der Lehrpersonen

- hohe Herausforderungen durch komplexe (gerade noch bewältigbare) Aufgabenstellungen

- Transparenz gegenüber den Lernenden hinsichtlich der Erwartungen

- Geben von ,guten' (entwicklungsfördernden, detaillierten) Rückmeldungen

Hattie und Yates (2014: 67) weisen ebenfalls darauf hin, dass Lernaufgaben insbesondere dann lernförderlich sind, wenn ihr Anforderungsniveau an der Schwelle zur Überforderung liegt, aber diese gerade noch bewältigbar sind (dies entspricht Vygotskys „Zone der nächsten Entwicklung" - Zone of proximal development; vgl. Vygotski 1978: 88) und wenn ein möglichst unmittelbares und regelmäßiges Feedback über den jeweiligen Bearbeitungsstand gegeben wird. Die Autoren verweisen auf die konsequente Umsetzung dieser Grundprinzipien in Computerspielen und begründen damit deren besondere Attraktivität: Computerspiele sind so konzipiert, dass die jeweiligen Anforderungen über den derzeitigen Fähigkeiten bzw. an der Schwelle zur Überforderung der Spieler/innen liegen, außerdem bekommen diese regelmäßig und möglichst unmittelbar nach jeder Aktion ein Feedback über den jeweiligen Spielstand.

Die vorangegangenen Ausführungen beschreiben Qualitätsmerkmale und Ansprüche an das Anforderungsniveau von komplexen (kompetenzorientierten) Aufgabenstellungen, sie bieten aber keine Lösungsansätze für die erste der beiden aufgeworfenen Kernfragen an: Wie bestimmt man die Komplexität von Aufgabenstellungen? Dazu gibt es verschiedenste allgemeine Modelle, etwa die Bloom'sche Taxonomie von Lernzielen oder fachspezifische Modelle, wie die in den Bildungsstandards für Geographie der Deutschen Gesellschaft für Geographie definierten Anforderungsbereiche (DGfG 2014, $30 \mathrm{ff}$.).

Im Zuge der Entwicklungsbegleitung zur Einführung der Neuen Mittelschule (NMS) in Österreich wird vor allem das aus dem angloamerikanischen Raum stammende Modell „Depth of Knowledge (DOK) " nach Norman Webb (2007) herangezogen, um die Komplexität von Aufgabenstellungen zu bestimmen. Dabei werden vier Niveaus (levels) definiert (vgl. ebd.: $11 \mathrm{ff}$.). Nachstehend werden diese näher erläutert und mit möglichen Beispielen aus dem GWUnterricht (Sekundarstufe I und II) versehen.

- Level 1: Erinnerung \& Reproduktion (Recall \& Reproduction): Wiedergabe von Fakten, Informationen, Begriffen, Anwendung von Formeln etc. (z. B. „Lege mit Hilfe des Atlas eine Liste mit den Staaten Südamerikas an und nenne jeweils 2 wichtige Agrarprodukte der einzelnen Staaten.")

- Level 2: Fertigkeiten \& Konzepte (Skills \& Concepts): Konzepte anwenden, mehrere Arbeitsschritte hintereinander durchführen, mögliche Lösungen durchdenken, Informationen filtern etc. (z. B. „Lies den Zeitungsartikel über die Erfahrungen mit der City-Maut in Oslo durch. Bereite anschließend eine Pro/Kontra-Liste für den/die Bürgermeister/in deiner Stadt vor, welche ihn/sie über mögliche Vor- und Nachteile, Probleme und Chancen der Einführung einer City-Maut informiert.")

- Level 3: Strategisches Denken (Strategic Thinking): logisches und strategisches Denken entwickeln, Pläne und Konzepte entwerfen, mehrere Lösungsansätze abwägen/begründen etc. (z. B. „Schaut euch die ersten 30 Minuten des Films, Plastic Planet 'an. Überlegt anschließend in einer Kleingruppe, welche Maßnahmen zur Vermeidung von Mikroplastik möglich sind. Was kann jede/r Einzelne sofort beitragen? Welche Maßnahmen müssen auf staatlicher Ebene gesteuert werden?")

- Level 4: Erweitertes Denken (Extended Thinking): Informationen vernetzen und zueinander in Beziehung setzen, Lösungsmöglichkeiten entwickeln, anwenden und abwägen etc. (z.B. „Nachfolgende Tabellen und Grafiken informieren über die Entwicklung der Elektromobilität seit 2010. Ein Zeitungsartikel informiert über derzeit in Entwicklung befindliche Innovationen. Überlegt in der Kleingruppe, was das für die Zukunft der Mobilität bedeuten kann (Ausstattung, Infrastruktur, etc.). Ab- 
schließend verfasst einen Zeitungsartikel: Ihr seid Reporter/innen einer Tageszeitung im Jahr 2050 und beschreibt die Entwicklung der Mobilität in den letzten 40 Jahren.")

Das Komplexitätsmodell nach Norman Webb ist zur Einschätzung der Komplexität von Aufgabenstellungen m.E. gut geeignet. Es unterstützt die Planung von Aufgabenstellungen in zweierlei Hinsicht: Es gibt Hilfestellung bei der Entwicklung von Aufgabenstellungen auf verschiedenen Niveaus und es hilft dabei, von den immer noch weit verbreiteten, reinen Reproduktionsaufgaben vermehrt Abstand zu nehmen. Damit soll nicht argumentiert werden, dass einfachere Aufgaben (DOK-Niveau 1 und 2) keinen Platz im Unterricht haben, aber im Sinne eines kompetenzorientierten Unterrichts sollten diese - vor allem in höheren Jahrgangsstufen - zunehmend weniger Raum einnehmen. Aufgabenstellungen der niedrigeren $\mathrm{Ni}$ veaustufen 1 und 2 sind $\mathrm{m}$. E. vor allem deshalb immer noch sehr weit verbreitet, insbesondere in schriftlichen und mündlichen Leistungsfeststellungen, weil sie eine einfache, eindeutige und wenig aufwändige Rückmeldung ermöglichen: es geht meist um richtig / falsch, vollständig/unvollständig, vorhanden/nicht vorhanden etc. Wenn z. B. die Nachbarstaaten Österreichs überprüft werden sollen (Level 1), ist eine einfache und eindeutige Rückmeldung möglich (z. B. 6 von 8 Punkten weil nur sechs Staaten angeführt wurden). Derartige Leistungen können jedoch nicht als Belege von Kompetenz geltend gemacht werden. Wie aber kann Rückmeldung bei komplexeren Aufgabenstellungen der Levels 3 und 4 erfolgen? Hier gibt es meist keine einfachen bzw. eindeutigen Kriterien. Eine Rückmeldung mit Punkten, Prozenten etc. ist meist nicht möglich, bzw. sehr problematisch. Daher wird im folgenden Abschnitt auf die zweite, eingangs erwähnte Kernfrage eingegangen: Wie bewertet man komplexe Leistungen?

\section{Aufgabe bearbeitet - und dann? Möglich- keiten einer lernförderlichen Rückmeldung}

In diesem Abschnitt wird mit Hilfe eines konkreten Umsetzungsvorschlages auf entwicklungs- bzw. lernförderliche Rückmeldemöglichkeiten durch die Lehrperson eingegangen. Zunächst muss jedoch betont werden, dass in einem kompetenzorientierten Unterricht nicht nur einer qualitätsvollen und entwicklungsfördernden Rückmeldung durch die Lehrperson (Fremdeinschätzung) ein wichtiger Stellenwert zukommt, sondern auch der Selbststeuerung sowie der Selbsteinschätzung der eigenen Leistungen durch die Lernenden. Schüler/innen müssen durch entspre- chende Möglichkeiten im Rahmen des Unterrichts in der Lage sein, ihre eigenen Leistungen (Stärken und Schwächen) in Bezug auf die Ziele realistisch einzuschätzen (vgl. Steffens \& Höfer 2016: 40 f.). Hilfreich ist es auch, wenn Schüler/innen im Sinne der Metakognition (vgl. Borich 2014: 326) angehalten und befähigt werden, über ihr eigenes Lernen, ihre Lernfortschritte sowie -schwierigkeiten zu reflektieren und daraus die dementsprechenden Schlüsse für das weitere Vorgehen zu ziehen. Steffens und Höfer (2016: 92) merken diesbezüglich unter Hinweis auf Hattie an, dass Feedback von Lernenden an die Lehrperson hilft, das Lernen sichtbar zu machen, indem die Lernenden beschreiben, wie ihr momentaner Lernstand ist, wo und welche Fehler sie machen oder was sie verstanden haben bzw. noch nicht verstehen. Dies ist eine unabdingbare Voraussetzung für das Feedback der Lehrperson an die Lernenden, denn nur wenn man als Lehrperson eine klare Vorstellung über den derzeitigen Lernstand der Lernenden hat, ist effizientes Feedback möglich (Hattie 2012: 115). Earl (2003) streicht diese Beurteilung des jeweiligen Lernstandes unter dem Schlagwort „assessment as learning“ heraus und betont damit die Notwendigkeit einer formativen Rückmeldung, um weiteres Lernen zu ermöglichen.

Anknüpfend an die im vorangegangenen Abschnitt definierten Komplexitätsstufen von Aufgabenstellungen wird nun der zweiten Kernfrage nachgegangen: Wie bewertet man komplexe Leistungen? ${ }^{2}$ Im Sinne obiger Ausführungen ist eine aussagekräftige und entwicklungsförderliche Rückmeldung für komplexe Aufgaben der Niveaustufen 3 und 4 nach Webbs DOK-Modell notwendig. Dafür wird hier die von dem amerikanischen Bildungsforscher Robert Marzano (2006) entwickelte 4.0-Skala vorgeschlagen und nachstehend näher erläutert. Eine erste Darstellung findet sich in Tabelle 2. Die englischsprachige Beschreibung bezieht sich dabei auf den Vorschlag von Marzano (2006: 93), die deutschsprachige auf die im Rahmen der Neuen Mittelschule verwendete Wortwahl (Schlichtherle, Weißkopf-Prantner \& Westfall-Greiter 2012: 5).

Diese allgemein formulierte Grundstruktur muss für konkrete Themen im Unterricht adaptiert werden, indem für jede Stufe dieser Skala die erwarteten Leistungen möglichst exakt beschrieben werden. Auszugehen ist dabei zunächst von der Stufe 3.0: Diese stellt insofern die wichtigste Stufe dar, als hier das ,Wunschbild' formuliert wird: was sollen Schüler/innen am Ende eines Abschnittes wissen, verstehen und

\footnotetext{
In diesem Artikel wird ausschließlich auf eine formative (entwicklungsfördernde) Rückmeldung von Schüler/innen-Leistungen eingegangen. Die rechtlichen Aspekte (Leistungsbeurteilung im Sinne einer Notengebung) sind aus Platzgründen nicht Teil dieser Überlegungen.
} 
Tab. 2: Grundstruktur einer 4.0 Skala

4.0 Zielbild übertroffen (Students know (can do) it well enough to make connections that weren't taught).

3.0 Zielbild getroffen (Students know (can do) everything that was taught without making mistakes).

2.0 Zielbild teils getroffen (Students know (can do) all the easy parts, but don't know (can't do) the harder parts).

1.0 Zielbild teils getroffen, aber nur mit Hilfe (With help, students know (can do) some of what was taught).

tun können? Sobald das Zielbild klar definiert und verbal beschrieben ist, kann an den anderen Niveaus der Skala gearbeitet werden: Was wäre eine Leistung/ Kompetenz, die gehaltvoller ist, als was aufgrund des Unterrichts erwartetet werden könnte (4.0), wie unterscheiden sich einfachere und schwierigere Komponenten des Themas (2.0), was wären mögliche Hilfestellungen (z.B. vereinfachte Materialien), die es Schüler/innen ermöglichen, zumindest Teile des Zielbildes zu erreichen (1.0)?

Diese allgemeinen Formulierungen werden nachstehend in einem konkreten Beispiel aus dem GWUnterricht erläutert, welches im Anschluss an die Erarbeitung der Klimazonen der Erde eingesetzt werden kann. Wie bereits oben erwähnt, ist bei der Erstellung der Skala vom intendierten Zielbild auszugehen (3.0), in diesem Sinne sollte auch beim Lesen der Skala zunächst das Zielbild in Augenschein genommen werden, erst dann die Abstufungen nach oben und unten.

\section{Beispiel: Gewinn einer Reise}

Ihre Eltern machen bei einem Preisausschreiben mit und gewinnen den Hauptpreis: eine einwöchige Reise zu einem Ziel und zu einem Zeitpunkt freier Wahl für die gesamte Familie! Die maximalen Kosten für vier Reisende dürfen jedoch EUR 12.000,00 nicht übersteigen. Da Ihr Vater als Kellner im Gastgewerbe arbeitet, kann er nur in der Nebensaison Urlaub nehmen, der Zeitpunkt der Reise wird daher auf die letzte Novemberwoche festgelegt. Nun beginnen die Überlegungen, wohin die Reise gehen soll. Alle sind sich rasch einig, dass das Ziel außerhalb Europas liegen soll. Sie werden von den Eltern beauftragt, drei Vorschläge auszuarbeiten und diese in eine gewünschte Reihenfolge zu bringen. AuBerdem soll ein Vorschlag ausgearbeitet werden, der aufgrund der Vorgaben schlecht bzw. nicht umsetzbar ist. Beschreiben Sie die Vorschläge und lassen Sie dabei folgende Überlegungen einfließen:

- Aus welchen Quellen werden Informationen bezogen? Warum wurden diese ausgewählt?

- Nach welchen Kriterien werden Ziele ausgewählt?

\section{Tab. 3: 4.0 Skala zu einem Aufgabenbeispiel (Klimazonen)}

\begin{tabular}{|c|c|}
\hline $\begin{array}{l}.0 \\
\text { Zielbild } \\
\text { bertroffen) }\end{array}$ & $\begin{array}{l}\text { Es werden Überlegungen angestellt, die über das Zielbild hinausgehen und auch nicht Thema des Unter- } \\
\text { richts waren, z. B. } \\
\text { - Wie kann ich die Reise möglichst ökologisch gestalten? (ökologischer Fußabdruck etc.) } \\
\text { - Es werden Überlegungen hinsichtlich der politischen, sozialen oder menschenrechtlichen Situation der } \\
\text { jeweiligen Destination angestellt und diese in den Entscheidungsprozess einbezogen. } \\
\text { - Es werden Überlegungen hinsichtlich gesundheitlicher und rechtlicher Aspekte angestellt (z. B. Sind Imp- } \\
\text { fungen notwendig? Brauche ich ein Visum? Wenn ja, welche Vorlaufzeiten sind hier zu beachten? etc.) }\end{array}$ \\
\hline $\begin{array}{l}.0 \\
\text { zielbild } \\
\text { etroffen) }\end{array}$ & $\begin{array}{l}\text { - Vorschläge sind vollzählig vorhanden (drei mögliche, ein ,problematischer') } \\
\text { - Informationen werden aus verschiedenen Medien (Internet, Atlas, etc.) und verschiedenen Quellenar- } \\
\text { ten (Texte, Klimadiagramme, -karten, etc.) entnommen und entsprechend vernetzt. Es handelt sich da- } \\
\text { bei durchgängig um seriöse / verlässliche Medien und Quellen. Die Informationen sind den jeweiligen } \\
\text { Quellen zuordenbar. } \\
\text { - In den Texten wird jeweils begründet argumentiert, warum es sich um eine geeignete bzw. ungeeignete } \\
\text { Destination handelt sowie welche Möglichkeiten (Aktivitäten) es vor Ort gibt. } \\
\text { - Die Vorschläge sind nach den genannten Vorgaben realistisch umsetzbar. } \\
\text { - Die Vorschläge sind in eine Reihenfolge gebracht, diese wird begründet argumentiert. } \\
\text { - Eine grobe Kostenschätzung ist vorhanden und einigermaßen realistisch. }\end{array}$ \\
\hline $\begin{array}{l}.0 \\
\text { Zielbild } \\
\text { ils getrof- }\end{array}$ & $\begin{array}{l}\text { - Vorschläge sind entweder nicht vollständig vorhanden oder vollständig vorhanden, aber inhaltlich } \\
\text { mangelhaft bzw. nicht ausreichend argumentiert. } \\
\text { - Es werden nur wenige Medien und Quellen verwendet, die Informationen sind zumindest teilweise } \\
\text { nicht zuordenbar. Es werden auch unseriöse Quellen verwendet (z. B. Blogeinträge in Reiseblogs, etc.) } \\
\text { - Es gibt keine bzw. keine begründete Reihung der Vorschläge. Vorschläge sind zwar argumentiert und } \\
\text { grundsätzlich umsetzbar, aber nicht unter den gegebenen Umständen (z. B. Reise in die Antarktis (kli- } \\
\text { matisch möglich), aber nur } 1 \text { Woche Zeit (organisatorisch / zeitlich unmöglich). } \\
\text { - Eine Kostenschätzung ist nicht vorhanden bzw. völlig unrealistisch. }\end{array}$ \\
\hline $\begin{array}{l}1.0 \\
\text { (Zielbild } \\
\text { teils mit } \\
\text { Hilfe ge- } \\
\text { troffen) }\end{array}$ & $\begin{array}{l}\text { - Eine Ausarbeitung von Vorschlägen gelingt nur mit Hilfe der Lehrperson (entsprechende Materialien } \\
\text { werden zur Verfügung gestellt bzw. Schüler/innen finden entsprechende Informationen nur mit Hilfe } \\
\text { der Lehrperson). } \\
\text { - Die Lehrperson wird während des Ausarbeitungsprozesses mehrmals zur Unterstützung angefordert } \\
\text { bzw. wird diese angeboten, da ansonsten kein Arbeitsfortschritt festgestellt werden kann. }\end{array}$ \\
\hline
\end{tabular}


Tab. 4: Webb und Marzano im Überblick

\begin{tabular}{lll}
\hline & Depth of Knowlede (Webb 2007) & 4.0 Skala (Marzano 2006) \\
\hline Anspruch & Komplexitätsgrad & Bewertungskriterien \\
\hline Ziel & $\begin{array}{l}\text { Einschätzung des Komplexitätsgrades von Aufga- } \\
\text { benstellungen; Hilfe bei der Erstellung von Aufga- } \\
\text { ben auf verschiedenen Niveaustufen }\end{array}$ & $\begin{array}{l}\text { Hilfe zur Bewertung (Rückmeldung) von Leistungen } \\
\text { aus komplexen Aufgaben }\end{array}$ \\
\hline \multirow{2}{*}{$\begin{array}{ll}\text { Zeitpunkt } \\
\end{array}$} & $\begin{array}{l}\text { Vor dem Unterricht: Auswahl bzw. Erstellung von } \\
\text { Aufgaben }\end{array}$ & $\begin{array}{l}\text { Nach dem Unterricht: Bewertung der Leistungen bei } \\
\text { der Ausarbeitung von Kompetenzaufgaben (Aufga- } \\
\text { ben, welche in Webb-Bereiche 3 und 4 fallen) }\end{array}$ \\
\hline
\end{tabular}

(z. B. Anreisezeit, Klima, Aktivitäten vor Ort, Reise- und Aufenthaltskosten,...)

- Verfassen Sie einen kurzen Text (max. halbe Seite) mit den wesentlichen Vorzügen und ev. Nachteilen der jeweiligen Destination. Begründen Sie alle Entscheidungen!

Mögliche Überlegungen hinsichtlich einer Einschätzung der Leistung könnten in einer 4.0 Skala, wie in Tabelle 3 dargestellt, erfolgen:

\section{Zusammenschau und Ausblick}

Vorliegender Artikel versuchte zunächst, die Notwendigkeit einer veränderten Lern-, Aufgaben- und Rückmeldekultur zu begründen (Stichwort: OutputOrientierung von Bildungssystemen). Im Anschluss daran wurde definiert, was unter einer kompetenzorientierten Aufgabenstellung zu verstehen ist: Es handelt sich dabei um Aufgabenstellungen, die keine einfachen Reproduktionsleistungen einfordern, sondern vielmehr zum Vernetzen und Anwenden von Wissen anregen und idealerweise im Sinne von Vygotskis Lernzonenmodell herausfordernd, d.h. schwierig, aber gerade noch bewältigbar, gestaltet sind. Schließlich wurde durch Webbs Modell ,Depth of Knowledge, in Verbindung mit Marzanos 4.0 Skala ein Modell zu Selbst- und Fremdeinschätzung von komplexen Leistungen vorgestellt und durch ein konkretes Beispiel verdeutlicht.

Tabelle 4 zeigt eine zusammenfassende Gegenüberstellung der Verwertungsaspekte dieser beiden Konzepte (Tabelle adaptiert nach Westfall-Greiter \& Hofbauer 2016: 1).

Die am Beginn des Abschnittes 4 postulierte Forderung nach einer vermehrten Einbeziehung der Schüler/innen in die Einschätzung ihrer eigenen Leistungen (Stichworte: Selbstkonzept, Selbsteinschätzung) kann ebenfalls mit der 4.0 Skala umgesetzt werden: entsprechend formuliert und in die Hände der Schüler/innen gegeben kann diese dazu dienen, die geforderten Kriterien besser zu verstehen und eine tiefere bzw. detailliertere Ausarbeitung der Aufgabenstellung anzuregen.
Zusammenfassend wird festgehalten, dass komplexe, herausfordernde und transfer-anregende Aufgabenstellungen einen zentralen Stellenwert in einem kompetenzorientierten Unterricht einnehmen sollten. Dies stellt große Herausforderungen für die Lehrpersonen dar: Die Entwicklung von kompetenzorientierten Aufgaben, die Bestimmung von deren Komplexität und die Rückmeldung der erbrachten Leistungen ist aufwändig und gerade anfänglich auch sehr herausfordernd. Gerade deshalb wäre auch eine vermehrte Kooperation im jeweiligen Fachbereich, aber auch über die Fächergrenzen hinweg wünschenswert, um anwendungsfähiges und anschlussfähiges, im Sinne von Weinert intelligentes Wissen (handlungskompetentes Wissen) zu fordern und zu fördern.

\section{Literatur}

Aebli, H. (1980): Denken: das Ordnen des Tuns. Band 1: Kognitive Aspekte der Handlungstheorie. Stuttgart: Klett-Cotta.

Astleitner, H. (2007): Das Verhältnis von Wissenschaft und Praxis bei innerer Differenzierung im Unterricht: Das besondere Problem der Umsetzungshilfen. In: Gastager, A.,T. Hascher \& H. Schwetz (Hrsg.): Pädagogisches Handeln: Balancing zwischen Theorie und Praxis: Beiträge zur Wirksamkeitsforschung in pädagogisch-psychologischem Kontext (Bd). Landau: Verlag Empirische Pädagogik. S. 137-146.

Borich, G. (2014): Effective Teaching Methods. ResearchBased Practice (International ed. of 8th revised ed.). Upper Saddle River, New Jersey: Pearson.

Brophy, J. (2000): Teaching. http://www.ibe.unesco.org/ fileadmin/user_upload/archive/publications/EducationalPracticesSeriesPdf/prac01e.pdf (23.12.2012).

Deci, E. \& R. Ryan (2002): Overview of self-determination theory. An organsimic dialectic perspective. In: ebd. (Hrsg.): Handbook of Self-Determination Research. Rochester: University of Rochester Press. S. 3-33.

Deutsche Gesellschaft für Geographie DGfG (Hrsg., 2014): Bildungsstandards im Fach Geographie für den Mittleren Schulabschluss mit Aufgabenbeispielen (8., aktualisierte Auflage). Bonn: Selbstverlag Deutsche Gesellschaft für Geographie. 
Dörner, D. (1990): Die Logik des Misslingens: strategisches Denken in komplexen Situationen. Reinbek bei Hamburg: Rowohlt.

Earl, L. (2003): Assessment as Learning. Using Classroom Assessment to Maximize Student Learning. Thousand Oaks: Corwin Press.

Gottein, H.-P. (2016): Tun sie denn, was sie wissen? Hochschuldidaktische Überlegungen für eine kompetenzorientierte und handlungspsychologisch begründete Lernumgebung in der Ausbildung von Lehrerinnen und Lehrern. Bad Heilbrunn: Klinkhardt.

Hattie, J. (2009): Visible Learning: A Synthesis of Over 800 Meta-Analyses Relating to Achievement. London \& New York: Routledge.

Hattie, J. (2012): Visible learning for teachers: maximizing impact on learning. London \& New York: Routledge.

Hattie, J. \& G. Yates (2014): Visible Learning and the Science of How We Learn. New York: Routledge.

Helmke, A. (2009): Unterrichtsqualität und Lehrerprofessionalität: Diagnose, Evaluation und Verbesserung des Unterrichts. Seelze-Velber: Kallmeyer.

Institut für Qualitätsentwicklung (Hessisches Kultusministerium) (Hrsg., 2011): Hessischer Referenzrahmen Schulqualität. Qualitätsbereiche, Qualitätsdimensionen und Qualitätskriterien. http://lakk.sts-gym-marburg. bildung.hessen.de/grundlagenpapiere/Hessischer_Referenzrahmen_Schulqualitaet_HRS_.pdf (02.12.2016).

Keller, S. \& C. Reintjes (2016): Aufgaben als Schlüssel zur Kompetenz: eine Einleitung. In: ebd. (Hrsg.): Aufgaben als Schlüssel zur Kompetenz. Didaktische Herausforderungen, wissenschaftliche Zugänge und empirische Befunde. Münster / New York: Waxmann. S. 15-25.

Klieme, E., H. Avenarius, W. Blum, P. Döbrich, H. Gruber, M. Prenze \& K. Reiss (2007): Zur Entwicklung nationaler Bildungsstandards. Expertise. Berlin: Bundesministerium für Bildung und Forschung (BMBF). https://www.bmbf. de/pub/Bildungsforschung_Band_1.pdf (02.12.2016).

Marzano, R. (2006): Classroom Assessment \& Grading That Work. Alexandria, VA: ASCD.

Meyer, H. (2004): Was ist guter Unterricht? Berlin: Cornelsen Scriptor.

Meyer, H. (2010): Merkmale guten Unterrichts - Ein Kriterienmix. In: Jürgens, E. \& J. Standop (Hrsg.): Was ist „guter" Unterricht? Namhafte Expertinnen und Experten geben Antwort. Bad Heilbrunn: Klinkhardt. S. 159-174.

Parchmann, I. \& S. Bernholt (2016): Aufgaben als Brücken zwischen Lebenswelt und Fachunterricht. In: Keller, S. \& C. Reintjes (Hrsg.): Aufgaben als Schlüssel zur Kompetenz. Didaktische Herausforderungen, wissenschaftliche Zugänge und empirische Befunde. Münster/New York: Waxmann. S. 41-51.
Reinfried, S. (2016): Kompetenzorientierte Lernaufgaben - mehr als alter Wein in neuen Schläuchen? In: Geographie aktuell und Schule (223/38. Jahrgang). S. 4-14.

Renkl, A. (1994): Träges Wissen: die „unerklärliche“ Kluft zwischen Wissen und Handeln. Ludwig-MaximiliansUniv., Inst. fürPädagog. PsychologieundEmpir. Pädagogik.

Reusser, K. (2013): Aufgaben - das Substrat der Lerngelegenheiten im Unterricht. In: Profi-L, (3/13). S. 4-6.

Schlichtherle, B., V. Weißkopf-Prantner \& T. WestfallGreiter (2012). Kriterienorientierte Leistungsfeststellung mit der 4.0-Skala. Zentrum für lernende Schulen. http://www.nmsvernetzung.at/mod/page/view. php?q=marzano\&id=4397 (02.12.2016).

Steffens, U. \& D. Höfer (2016): Lernen nach Hattie. Wie gelingt guter Unterricht? Weinheim und Basel: Beltz.

Vygotski, L. (1978): Mind in Society: The Development of Higher Psychological Processes. Cambridge (Mass.): Harvard University Press.

Webb, N. (2007): Issues Related to Judging the Alignment of Curriculum Standards and Assessments. In: Applied Measurement in Education 20/1. S. 7-25.

Weinert, F. E. (2002): Leistungsmessungen in Schulen (2., unveränderte Auflage). Weinheim und Basel: Beltz.

Westfall-Greiter, T. \& C. Hofbauer (2016): Webb und Marzano: 4 ist nicht gleich 4.0. Zentrum für lernende Schulen. http://www.nmsvernetzung.at/mod/page/view. php?q=marzano\&id=4397 (02.12.2016).

Whitehead, A. N. (1967): The Aims of Education. In: ebd. (Hrsg.): The Aims of Education and Other Essays. New York: MacMillan, 1-14. In: Riffert, F. (2008): VÜ: Wissenschaftstheoretische Diskussion pädagogischer Themen WS 2008/09. Salzburg: Universität Salzburg. S. 13-26.

Wilkening, M. (2016): Praxisbuch Feedback im Unterricht. Lernprozesse reflektieren und unterstützen. Weinheim / Basel: Beltz.

Winterton, J., F. Delamare-LeDeist \&E. Stringfellow(2006): Typology of knowledge, skills and competences: clarification of the concept and prototype. Luxembourg: Office for Official Publications of the European Communities.

Wormeli, R. (2006): Fair Isn't Always Equal. Assessing and Grading in the Differentiated Classroom. Portland (Maine): Stenhouse Publishers.

Ziegler, E., E. Stern \& A. Neubauer (2012): Kompetenzen aus der Perspektive der Kognitionswissenschaften und der Lehr-Lern-Forschung. In: Paechter M. et al. (Hrsg.): Handbuch Kompetenzorientierter Unterricht. Weinheim und Basel: Beltz. S. 14-26. 